\title{
Self-Similar Perturbation Theory
}

\author{
V. I. Yukalov ${ }^{1,2}$ and E. P. Yukalova ${ }^{2,3}$ \\ ${ }^{1}$ Bogolubov Laboratory of Theoretical Physics \\ Joint Institute for Nuclear Research, Dubna 141980, Russia \\ ${ }^{2}$ Instituto de Fisica de São Carlos, Universidade de São Paulo \\ Caixa Postal 369, São Carlos, São Paulo 13560-970, Brazil \\ ${ }^{3}$ Department of Computational Physics \\ Laboratory of Computing Techniques and Automation \\ Joint Institute for Nuclear Research, Dubna 141980, Russia
}

\begin{abstract}
A method is suggested for treating those complicated physical problems for which exact solutions are not known but a few approximation terms of a calculational algorithm can be derived. The method permits one to answer the following rather delicate questions: What can be said about the convergence of the calculational procedure when only a few its terms are available and how to decide which of the initial approximations of the perturbative algorithm is better, when several such initial approximations are possible? Definite answers to these important questions become possible by employing the self-similar perturbation theory. The novelty of this paper is in developing the stability analysis based on the method of multipliers and in illustrating the efficiency of this analysis by different quantum-mechanical problems.
\end{abstract}

PACS: 02.30.Mv, 03.65.Ge, 11.15.Bt, 11.15.Tk 
Running head: Self-Similar Perturbation Theory

Number of pages: 45

Number of Tables: 2

\section{Mailing addresses:}

till October 10, 1999:

Prof. V.I. Yukalov

Instituto de Fisica de Sao Carlos

Universidade de Sao Paulo

Caixa Postal 369, Sao Carlos

Sao Paulo 13560-970, Brazil

Phone: 55 (16) 271-2012

Fax: 55 (16) 271-3616

E-mail: yukalov@if.sc.usp.br

after October 10, 1999:

Prof. V.I. Yukalov

Bogolubov Laboratory of Theoretical Physics

Joint Institute for Nuclear Research

Dubna 141980, Russia

E-mail: yukalov@thsun1.jinr.ru

Tel: 7 (096) 216-3947 (office); 7 (096) 213-3824 (home)

Fax: 7 (096) 216-5084 


\section{Introduction}

Realistic physical problems can practically never be solved exactly, quantum field theory being among these the most difficult problems. Then one has to resort to some approximations. One such a reliable approximation is the Gaussian-effective-potential approach [1-5]. This approach contains the leading $1 / N$ result as its formal $N \rightarrow \infty$ limit and also the one-loop result as its formal $\hbar \rightarrow 0$ limit. Thus, it encompasses both of the other popular approaches to effective potentials, transforming their leading-order expansions and displaying a much richer structure $[5,6]$. At zero temperature, the Gaussian effective potential can be described as a variational approximation to the vacuum energy density constructed with the Gaussian trial wave functions shifted by a constant classical background field [7]. The finite-temperature Gaussian effective potential is just the approximate free energy in the self-consistent harmonic approximation $[7,8]$. In this way, the Gaussian effective potential in quantum field theory is a direct analog of the variational ground-state energy in quantum mechanics, so that the quantum-mechanical language can be directly transcribed to the quantum-field-theory language [1]. The difference is that variational methods in quantum mechanics permit one to derive several nice comparison theorems [9-13] for the ground-state energy, which is rather difficult, if possible, in quantum field theory.

To obtain corrections to a chosen initial approximation, one has to develop some perturbative algorithm about the given approximation. Thus post-Gaussian corrections to the Gaussian effective potential may be obtained [14,15], allowing the variational parameter to change from one order to the next for the expansion to yield convergent results [14-17]. In the same spirit, one can calculate the effective potentials or energies as well as wave functions $[18,19]$. Different variants of such an approach are called in literature by various names, as modified perturbation theory, renormalized perturbation theory, optimized perturbation theory, controlled perturbation theory, oscillator-representation method, and so on [20-31]. One often dignifies this kind of methods as nonperturbative, implying that they result in modified, or resummed, expansions having a more complicated 
structure than simple weak-coupling power series. However, the term "nonperturbative" is, to our mind, not only cumbersome but somewhat misleading. This is because from the mathematical point of view any regular procedure producing a sequence of approximations is perturbation theory, irrespectively of what initial approximation is chosen and what additional conditions in the course of calculation are imposed. The role of such additional conditions is to reorganize, by defining control functions, a given series into another one with better convergence properties [20-22], which is necessary because of the divergence of standard weak-coupling power series [32]. Note that the renormalization scale, appearing in perturbative expansions for observable quantities of field theories, can also be treated as a control function that may be defined by some additional conditions $[27,28,33]$ or invoking information from experiment $[33,34]$.

The convergence of modified perturbation theory can be treated in three ways. The straightforward case is when the model considered is simple enough to permit the evaluation of perturbative terms of arbitrary large orders. This happens for zero- and onedimensional anharmonic oscillators [35-37]. But clearly one cannot expect to have the same luck for less trivial models.

Another possibility to check convergence is when the exact or very accurate numerical solution of the problem is available. This is so for many quantum-mechanical problems. Then one may directly compare the calculated perturbative terms with the known accurate solutions. If these terms approach the latter, one tells that the perturbation procedure is convergent. Since in this case one deals with a finite number of approximate terms, it is more correct to say that one observes numerical convergence or local convergence. It is this type of convergence that one deals with in any calculational procedure stopping at a finite-order approximation. Certainly, the luxury of possessing for comparison exact or almost exact numerical results is rather rare for realistic physical problems.

The numerical or local convergence may be also observed when one is able to find quite a number of perturbative terms, say several tens or hundreds of them, so that the calculational procedure saturates at some value that changes less and less with the increasing order of approximation. Again, it is not too realistic to hope to reach such a 
saturation with tens or hundreds of perturbative terms in complicated problems, such as field theory, usually allowing just a few terms.

What then can be said about the reliability of the results obtained by means of a perturbation algorithm, even if this is an optimized or modified perturbation theory, when one has in hands only a few terms and no exact answers are known? It may happen that the subsequent perturbative results quantitatively are not close to each other, then which of them is preferable? For example, optimized perturbation theory has been systematically tested using different ansatze for a range of different physical quantities in quantum electrodynamics and quantum chromodynamics [33]. Although in some specific applications this theory has met with success, in the majority of tests the optimization procedure was not successful. Typically, though the sign of the coefficients are correctly predicted, the optimization ansatze give values that are an order of magnitude too large or too small. Thus one comes to the conclusion that it is difficult to be optimistic about the general usefulness of optimized perturbation theory [33]. Even the more dramatic situation is when the neighbour-order results differ qualitatively, thus yielding different physics. For instance, for $(2+1)$-dimensional scalar theory, the analysis of the Gaussian effective potential shows a first-order phase transition, in contrast with the post-Gaussian approximation indicating a phase transition of second order [15]. Another example is the so-called autonomous version of the scalar field theory, which exists in the Gaussian approximation but does not survive, becoming unstable, in the higher orders of the postGaussian expansion [15].

Thus, for any calculational algorithm, including all variants of modified or optimized perturbation theory, there exists the general question: "How can one trust to a perturbative procedure when only a few first approximations are available and neither exact solutions, nor accurate numerical calculations, nor detailed experiments are known for comparison?" To say this in other words: "Is it possible to formulate a calculational algorithm that would be equipped by an internal self--consistent criterion allowing to estimate the validity of the obtained approximations?" In the present paper we aim at suggesting a positive answer to this question. The novelty of this paper is twofold: First, we de- 
velop the stability analysis, based on the method of multipliers, so that it really becomes possible to check the validity of the calculated approximations. Second, we illustrate the efficiency of the developed analysis by a number of quantum-mechanical problems. Among the latter, we consider the zero-dimensional $\varphi^{4}$ theory and anharmonic oscillators with integer powers. Although these models have been treated earlier by the self-similar approximation theory, however the stability analysis, as developed in this paper, have not been applied to them. The main part of the paper is devoted to the problems that have not yet been treated by the self-similar perturbation theory. These are the Hamiltonians with power-law potentials, having arbitrary noninteger powers, and a Hamiltonian with a logarithmic potential. In this way, the results presented in the paper are new.

\section{Basic Formulas}

Our consideration is based on the self-similar approximation theory [38-45]. This approach is more general than modified perturbation theory because of the possibility to reformulate calculational procedure as the problem of analyzing the evolution equation

for a controlled dynamical system, thus, permitting us to employ powerful techniques of dynamical theory and optimal control theory. We shall not repeat here the mathematical foundations for the self-similar approximation theory, which have been expounded in detail in earlier papers [38-45]. We will only mention the main idea of the approach and delineate its scheme, necessary for the following illustrations; but we shall concentrate on the stability problem that has not yet been considered carefully, the problem which is pivotal for the motivation of this paper, and which would make it possible to answer the questions formulated in the Introduction.

Assume that we are looking for a function $f(x)$ of a variable $x$. For simplicity, we imply that the function and variable are real, though the whole procedure can be straightforwardly extended to the case of complex functions and variables. Let the sought function $f(x)$ cannot be found exactly but only its perturbative expressions $p_{k}(x)$, with $k=0,1,2, \ldots$, can be obtained in the asymptotic vicinity of some point, say $x=0$. That 
is,

$$
f(x) \simeq p_{k}(x) \quad(x \rightarrow 0)
$$

The standard form of $p_{k}(x)$ is a series in powers of $x$, because of which $x$ is called the expansion parameter. This can be, e.g., a coupling parameter. Such series are practically always divergent. If a number of terms $p_{k}(x)$, about tens or more, were known, one could invoke some resummation techniques for ascribing a meaningful value to a divergent series [46]. But these techniques are useless when only a few perturbative terms are available. With the knowledge of just a few terms, one could find the limit of a sequence if a recurrence relation between subsequent terms were known. However, such recurrent relations are not merely difficult to discover but they have sense only for convergent sequences. Really, for an asymptotic series $p_{k}(x)$, meaningful solely for asymptotically small $x \rightarrow 0$ and diverging for any finite $x$, a recurrence relation between the terms $p_{k}(x)$ and $p_{k+1}(x)$ cannot be defined since the limit of $p_{k}(x)$, as $k \rightarrow \infty$, does not exist. Nevertheless, if the sequence of approximations $\left\{p_{k}(x)\right\}$ is obtained from one given system of equations, corresponding to a physical problem under consideration, by means of the same perturbative algorithm, the set of found terms $p_{k}(x)$ does contain information about the sought function $f(x)$. But this information, because of divergence of the sequence $\left\{p_{k}(x)\right\}$, is hidden, or we can say that it is enciphered, encoded.

The first thing we have to do in order to decipher, to decode the hidden information is to reorganize the sequence $\left\{p_{k}(x)\right\}$ to another sequence that would be convergent at finite $x$. In the process of this reorganization, or renormalization, that can be symbolically denoted as

$$
\mathcal{U}\left\{p_{k}(x)\right\}=\left\{F_{k}(x, u)\right\}
$$

we introduce auxiliary functions $u=u_{k}(x)$ so that the sequence $\left\{f_{k}(x)\right\}$ of the terms

$$
f_{k}(x) \equiv F_{k}\left(x, u_{k}(x)\right)
$$

be convergent [20]. Because of the role of the functions $u_{k}(x)$ in controlling convergence, they can be named the control functions. For a convergent sequence $\left\{f_{k}(x)\right\}$ a limit $f^{*}(x)$, as $k \rightarrow \infty$, exists, which represents the sought function $f(x)$. Now the problem of finding 
a recurrence relation, or mapping, between subsequent terms $f_{k}(x)$ and $f_{k+1}(x)$ can be meaningful.

The main idea in finding a relation between the terms of a sequence $\left\{f_{k}(x)\right\}$ is to try to construct a dynamical system with discrete time, being the approximant number $k=0,1,2, \ldots$, so that the motion from $f_{k}(x)$ to $f_{k+n}(x)$ would correspond to the evolution of this dynamical system. For this purpose, we need, first, to pass from the sequence $\left\{f_{k}(x)\right\}$ to a set of endomorphisms, which is done as follows. Define the expansion function $x_{k}(\varphi)$ by the equation

$$
F_{0}\left(x, u_{k}(x)\right)=\varphi, \quad x=x_{k}(\varphi)
$$

Introduce an endomorphism $y_{k}$ by the transformation

$$
y_{k}(\varphi) \equiv f_{k}\left(x_{k}(\varphi)\right)
$$

where, by definition $(4), y_{0}(\varphi)=\varphi$. As is evident, the sequences $\left\{y_{k}(\varphi)\right\}$ and $\left\{f_{k}(x)\right\}$ are bijective, and the limit $y^{*}(\varphi)$ of the former sequence is in one-to-one correspondence with the limit $f^{*}(x)$ of the latter sequence. The limit $y^{*}(\varphi)$ of the sequence of endomorphisms $\left\{y_{k}(\varphi)\right\}$ is nothing but a fixed point for which

$$
y_{k}\left(y^{*}(\varphi)\right)=y^{*}(\varphi)
$$

A family of endomorphisms in the vicinity of a fixed point satisfies the equation

$$
y_{k+n}(\varphi)=y_{k}\left(y_{n}(\varphi)\right)
$$

which can be easily shown by checking that, for $\varphi \rightarrow y^{*}$, Eq. (7) reduces to the identity $y^{*}=y^{*}$. In physical parlance, Eq. (7) is termed the property of self-similarity, the usage of which justifies the adjective self-similar, as is employed with respect to perturbation theory in the title of this paper. In the mathematical language, the equations $y_{k+n}=y_{k} \cdot y_{n}$ and $y_{0}=1$ are the semigroup properties. A family of endomorphisms $\left\{y_{k} \mid k=0,1,2, \ldots\right\}$, equipped with the semigroup properties, forms a cascade, that is, a dynamical system with discrete time. Since, by construction, the cascade trajectory $\left\{y_{k}(\varphi)\right\}$ is bijective to the approximation sequence $\left\{f_{k}(x)\right\}$, we call $\left\{y_{k} \mid k=0,1,2, \ldots\right\}$ the approximation cascade. 
To consider time evolution, it is convenient to deal with continuous time. For this, we may embed the cascade into a flow,

$$
\left\{y_{k} \mid k=0,1,2, \ldots\right\} \in\left\{y_{t} \mid t \geq 0\right\}
$$

which implies that the flow trajectory passes through all points of the cascade trajectory,

$$
y_{t}(\varphi)=y_{k}(\varphi) \quad(t=k=0,1,2, \ldots)
$$

and the same semigroup property, as for the cascade, is true for the flow,

$$
y_{t+\tau}(\varphi)=y_{t}\left(y_{\tau}(\varphi)\right)
$$

The flow $\left\{y_{t} \mid t \geq 0\right\}$ embedding the approximation cascade is called the approximation flow.

From the group relation (10), the Lie equation

$$
\frac{\partial}{\partial t} y_{t}(\varphi)=v\left(y_{t}(\varphi)\right)
$$

immediately follows, in which $v(y)$ is a velocity field. We need to find a fixed point $y^{*}(\varphi)$ of the approximation flow whose motion is given by the evolution equation (11). Consider the latter starting from the point $y_{k}(\varphi)$ at $t=k$ and moving to an approximate expression for the fixed point $y_{k+1}^{*}(\varphi, \tau) \equiv y_{k+\tau}^{*}(\varphi)$ occurring at the time $t=k+\tau$. Then, integrating Eq. (11), we come to the evolution integral

$$
\int_{y_{k}}^{y_{k+1}^{*}} \frac{d y}{v(y)}=\tau
$$

in which $y_{k+1}^{*}=y_{k+1}^{*}(\varphi, \tau)$ and $y_{k}=y_{k}(\varphi)$.

To make the evolution integral (12) useful, we have to concretize the flow velocity. The latter can be done by the Euler discretization giving for the velocity $v\left(y_{k}(\varphi)\right) \equiv v_{k}(\varphi)$ the form

$$
v_{k}(\varphi)=F_{k+1}\left(x, u_{k}\right)-F_{k}\left(x, u_{k}\right)+\left(u_{k+1}-u_{k}\right) \frac{\partial}{\partial u_{k}} F_{k}\left(x, u_{k}\right),
$$

where $x=x_{k}(\varphi)$ and $u_{k}=u_{k}\left(x_{k}(\varphi)\right)$. The discretized velocity (13) may be termed the cascade velocity. To obtain an explicit expression for the latter, we need yet to define 
the control functions $u_{k}(x)$. This can be realized being based on the following reasoning. As is clear from the Lie evolution equation (11), at the fixed point $y^{*}$, when $y_{t}\left(y^{*}\right)=y^{*}$, the flow velocity is zero, $v\left(y^{*}\right)=0$. Hence, closer we are to a fixed point, smaller is the absolute value of velocity. This suggests that the fastest convergence to the fixed point is achieved if control functions provide the minimum of the velocity modulus

$$
\left|v_{k}(\varphi)\right| \leq\left|F_{k+1}\left(x, u_{k}\right)-F_{k}\left(x, u_{k}\right)\right|+\left|\left(u_{k+1}-u_{k}\right) \frac{\partial}{\partial u_{k}} F_{k}\left(x, u_{k}\right)\right| .
$$

To minimize the second term in the right-hand side of the latter inequality, we may set

$$
\left(u_{k+1}-u_{k}\right) \frac{\partial}{\partial u_{k}} F_{k}\left(x, u_{k}\right)=0
$$

The principal usage of Eq. (15) is as follows. We are looking for a solution of the equation

$$
\frac{\partial}{\partial u_{k}} F_{k}\left(x, u_{k}\right)=0, \quad u_{k+1} \neq u_{k}
$$

which is labelled as the principal of minimal sensitivity [27], and which defines the control function $u_{k}(x)$. If the latter equation has no solution, we put $u_{k+1}=u_{k}$. If this equation possesses several physically meaningful solutions, we must take that of them which minimizes the cascade velocity (14). In the other way, we could set to zero the first term in the right-hand side of Eq. (14), thus coming to the principle of minimal difference [20]. But then we should invoke some additional condition for defining $u_{k+1}$. In what follows, we use the fixed-point condition (15), so that the cascade velocity (13) becomes

$$
v_{k}(\varphi)=F_{k+1}\left(x, u_{k}\right)-F_{k}\left(x, u_{k}\right)
$$

where $x=x_{k}(\varphi)$ and $u_{k}=u_{k}\left(x_{k}(\varphi)\right)$.

In this way, taking into account Eqs. (3)-(5), we rewrite the evolution integral (12) in the form

$$
\int_{f_{k}}^{f_{k+1}^{*}} \frac{d \varphi}{v_{k}(\varphi)}=\tau
$$

in which $f_{k}^{*}=f_{k}^{*}(x, \tau)$ is a $k$-order self-similar approximant for the sought function $f(x)$ and $f_{k}=f_{k}(x)$. The parameter $\tau$ in Eq. (12) is an effective minimal time necessary for reaching a fixed point $y_{k+1}^{*}$. If no constraints are imposed on the properties of the sought 
function, then the minimal time is the minimal number of steps, that is $\tau=1$. When some additional conditions are imposed, which the sought function has to satisfy, then $\tau=\tau_{k}(x)$ is to be treated as another type of control functions defined by these conditions. One more possibility of fixing $\tau$ is connected with the problem of stability, as is described below.

The problem of stability is extremely important. This is just what permits us to decide which of the found self-similar approximants are trustworthy and which of them are better then others. Let a set of mappings $\left\{y_{k}(\varphi)\right\}$ be given. Stability analysis is based on the concept of the local mapping multipliers

$$
\mu_{k}(\varphi) \equiv \frac{\partial}{\partial \varphi} y_{k}(\varphi)
$$

When

$$
\left|\mu_{k}(\varphi)\right|<1
$$

for a fixed $k$, one says that the mapping at a $k$-step is locally stable with respect to the initial point $\varphi$. If $\left|\mu_{k}(\varphi)\right|=1$, one tells that the mapping is locally neutral. In our case, the sequence $\left\{y_{k}(\varphi)\right\}$ is the trajectory of the approximation cascade. Therefore, if inequality (19) holds true, we should tell that the cascade at a $k$-step, or the trajectory at a $k$-point, is locally stable with respect to the initial point $\varphi$. Similarly, by means of the ultralocal multiplier

$$
\bar{\mu}_{k}(\varphi) \equiv \frac{\delta y_{k}(\varphi)}{\delta y_{k-1}(\varphi)}=\frac{\mu_{k}(\varphi)}{\mu_{k-1}(\varphi)}
$$

one may characterize the local stability with respect to the variation of the value $y_{k-1}$ preceeding the point $y_{k}$, which takes place for

$$
\left|\bar{\mu}_{k}(\varphi)\right|<1
$$

For brevity, we shall say that the cascade at a $k$-step is locally stable if inequality (19) is satisfied and it is ultralocally stable when condition (21) is valid. The latter can also be called the contraction condition. The images of the corresponding multipliers for the domain of the variable $x$ are defined as

$$
m_{k}(x) \equiv \mu_{k}\left(F_{0}\left(x, u_{k}(x)\right)\right), \quad \bar{m}_{k}(x) \equiv \bar{\mu}_{k}\left(F_{0}\left(x, u_{k}(x)\right)\right)
$$


in accordance with Eq. (4). Note that the local multipliers (18) define the local Lyapunov exponents

$$
\lambda_{k}(\varphi) \equiv \frac{1}{k} \ln \left|\mu_{k}(\varphi)\right| \quad(k=1,2, \ldots),
$$

so that the stability condition (19) is equivalent to $\lambda_{k}(\varphi)<0$. Some more information on dynamical systems and their stability can be found in Refs. [47-50].

The local stability of an approximation cascade $\left\{y_{k}\right\}$, that is, the local stability of its trajectory $\left\{y_{k}(\varphi)\right\}$, means the local convergence of the approximation sequence $\left\{f_{k}(x)\right\}$, bijective to this trajectory. In other words, if Eq. (19) is correct, then the $k$-order approximation is closer to a fixed point, that is more accurate, than the initial approximation. And when Eq. (21) is valid, the $k$-order approximation is better than the $(k-1)$-order approximation. The stability conditions (19) and (21) are sufficient for local convergence, but not necessary. So, when such conditions do not hold, this does not necessarily mean local divergence, but only tells that convergence cannot be guaranteed.

In order to characterize the type of convergence, let us consider the variation

$$
\delta y_{k}(\varphi) \equiv y_{k}(\varphi+\delta \varphi)-y_{k}(\varphi)=\mu_{k}(\varphi) \delta \varphi .
$$

Because of the relation (23) between the local multipliers and Lyapunov exponents, we have

$$
\left|\delta y_{k}(\varphi)\right|=|\delta \varphi| \exp \left\{\lambda_{k}(\varphi) k\right\} .
$$

For the stable process, when $\lambda_{k}(\varphi)<0$, the value $\left|\delta y_{k}(\varphi)\right|$ exponentially decreases with $k$. Therefore one tells that the calculational procedure is exponentially stable [51]. This is equivalent to saying that the approximation sequence $\left\{f_{k}(x)\right\}$ exponentially converges, since this sequence is, by construction, bijective to the approximation cascade trajectory $\left\{y_{k}(\varphi)\right\}$. Similarly, we could consider the variation

$$
\delta y_{k}^{*}(\varphi) \equiv y_{k}^{*}(\varphi+\delta \varphi)-y_{k}^{*}(\varphi)=\frac{\delta y_{k}^{*}(\varphi)}{\delta y_{k}(\varphi)} \mu_{k}(\varphi) \delta \varphi,
$$

which results in the expression

$$
\left|\delta y_{k}^{*}(\varphi)\right| \leq C|\delta \varphi| \exp \left\{\lambda_{k}(\varphi) k\right\}
$$


provided that $\left|\delta y_{k}^{*}(\varphi) / \delta y_{k}(\varphi)\right| \leq C$. The latter inequality holds true for the stable process, when $\lambda_{k}(\varphi)<0$, then $v_{k}(\varphi) \rightarrow 0$, as $k \rightarrow \infty$, and $y_{k}(\varphi) \rightarrow y_{k}^{*}(\varphi)$, so that $\left|\delta y_{k}^{*}(\varphi) / \delta y_{k}(\varphi)\right| \rightarrow 1$.

When the values of the sought function $f(x)$ can be found numerically, the accuracy of our calculational procedure can be characterized by the relative errors

$$
\varepsilon_{k}(x) \equiv \frac{f_{k}(x)-f(x)}{|f(x)|} \times 100 \%, \quad \varepsilon_{k}^{*}(x) \equiv \frac{f_{k}^{*}(x)-f(x)}{|f(x)|} \times 100 \% \text {. }
$$

Analysing these, we can judge whether the accuracy of the procedure becomes higher with increasing approximation order $k$. But if $f(x)$ is not known, how could we decide about the validity of approximations? In such a case, we have to be guided by the stability analysis dictating the following strategy: (i) When both stability conditions (19) and (21) hold true, then we can be certain that we are approaching the correct answer. In this case, the minimal time $\tau$ in the evolution integral (17) corresponds to the minimal number of steps, that is to $\tau=1$, necessary for reaching $f_{k+1}^{*}$ starting from $f_{k}$. (ii) When condition (19) holds but (21) does not, this means that the $k$-order approximation is certainly better than the initial approximation but may be worse than the preceeding $(k-1)-$ order approximation. In this situation, the accuracy of the sought approximation can be improved by employing the middle-point method, well known in numerical analysis [51]. The middle point can be defined in two ways, explicit and implicit. For the evolution integral (17), the explicit definition of the middle point implies that we have to associate $f_{k+1}^{*}$ with the middle of the interval between $k$ and $k+1$, that is, we have to set $\tau=\frac{1}{2}$. The implicit definition of the middle point between two approximations, say $F_{k+1}$ and $F_{k}$, is to take the average $\frac{1}{2}\left(F_{k+1}+F_{k}\right)$ of these approximations [51]. Using this average, instead of $F_{k+1}$, in the cascade velocity (16) yields the factor $\frac{1}{2}$ for the latter. This evidently is identical to putting $\tau=\frac{1}{2}$ in the evolution integral (17). So, the explicit and implicit ways of defining the middle point are equivalent to each other. (iii) Finally, if both conditions (19) and (21) are not valid, we cannot trust to the result of calculations. This can happen if the choice of control functions or the initial approximation, or both of them are not appropriate. Then we need to change these so that to achieve the stability 
of the procedure.

One more question could be as follows. Assume that we can realize our calculational procedure in two ways, say using different initial approximations. Then which of these ways should we prefer? The answer to this question straightforwardly follows from the stability analysis: That of several possible ways is preferable which is the most stable.

Thus, in the method of self-similar approximants, there is a unique opportunity to control the validity of calculations not knowing the sought function and having a small number of terms. In order to unambiguously prove all this, we consider in the following sections several examples for which numerical results are available. Comparing the latter with our predictions, everyone can directly observe that the method does work.

\section{Effective Potentials}

Consider the case of the so-called zero-dimensional $\varphi^{4}$ theory. The effective potential, or generating functional, has the structure

$$
f(g)=-\ln Z(g), \quad Z(g)=\frac{1}{\sqrt{\pi}} \int \exp \{-S(g, \varphi)\} d \varphi
$$

with the action

$$
S(g, \varphi)=\varphi^{2}+g \varphi^{4}
$$

where the field $\varphi$ is a real variable, $\varphi \in \mathbf{R}$, and the coupling parameter $g \in[0, \infty)$. Expanding $f(g)$ in powers of $g$ yields, as is well known, divergent series.

To introduce control functions, we rewrite the action (27) as

$$
\begin{gathered}
S(g, \varphi)=S_{0}(\varphi, u)+\Delta S(g, \varphi, u) \\
S_{0}(\varphi, u)=u^{2} \varphi^{2}, \quad \Delta S(g, \varphi, u)=\left(1-u^{2}\right) \varphi^{2}+g \varphi^{4} .
\end{gathered}
$$

When $\Delta S=0$, we have, in the place of Eq. (26),

$$
F_{0}(g, u)=\ln u
$$


Invoking the expansion in powers of $\Delta S$, we find

$$
F_{k}(g, u)=F_{k-1}(g, u)+\sum_{p=0}^{k} c_{k p} \alpha^{k-p} \beta^{p}
$$

with the notation

$$
\alpha=\alpha(u) \equiv 1-\frac{1}{u^{2}}, \quad \beta=\beta(u) \equiv \frac{3 g}{u^{4}}
$$

and the coefficients

$$
\begin{gathered}
c_{10}=-\frac{1}{2}, \quad c_{11}=\frac{1}{4}, \quad c_{20}=-\frac{1}{4}, \quad c_{21}=\frac{1}{2}, \quad c_{22}=-\frac{1}{3}, \\
c_{30}=-\frac{1}{6}, \quad c_{31}=\frac{3}{4}, \quad c_{32}=-\frac{4}{3}, \quad c_{33}=\frac{11}{12}, \\
c_{40}=-\frac{1}{8}, \quad c_{41}=1, \quad c_{42}=-\frac{10}{3}, \quad c_{43}=\frac{11}{2}, \quad c_{44}=-\frac{34}{9},
\end{gathered}
$$

and so on. As is seen, expression (30) is not a series in powers of $g$ but a bipolynomial with respect to the variables $\alpha$ and $\beta$ defined in Eq. (31).

Control functions $u_{k}(g)$ are given by the fixed-point condition (15). This means that for odd $k=2 n+1$, we use the equation $\partial F_{k} / \partial u_{k}=0$, while for even $k=2 n$, for which the latter equation has no solution, we put $u_{k}=u_{k-1}$. This procedure gives

$$
u_{k}(g)=\left[\frac{1}{2}\left(1+\sqrt{1+12 s_{k} g}\right)\right]^{1 / 2},
$$

where $s_{1}=s_{2}=1, s_{3}=s_{4}=2.239674, \ldots$ For large $k \gg 1$, one has $s_{k} \sim k^{\varepsilon}$, with $1 \leq \varepsilon \leq 2$. Substituting $u_{k}(g)$ into Eq. (31), we have the relation

$$
\alpha\left(u_{k}(g)\right) \equiv \alpha_{k}(g)=s_{k} \beta\left(u_{k}(g)\right)
$$

in which

$$
\alpha_{k}(g)=1+\frac{1-\sqrt{1+12 s_{k} g}}{6 s_{k} g} .
$$

To construct the approximation cascade, we directly follow the procedure of Sec. 2 . The sole unimportant difference is that, instead of the variable $x$ there, we have here the variable $g$. The equation (4) for the expansion function now reads $\ln u_{k}\left(g_{k}(\varphi)\right)=\varphi$, which yields

$$
g_{k}(\varphi)=\frac{e^{2 \varphi}}{3 s_{k}}\left(e^{2 \varphi}-1\right)
$$


The transformation (5) takes the form

$$
y_{k}(\varphi)=\varphi+\sum_{p=1}^{k} A_{k p} \alpha^{p}(\varphi)
$$

where the coefficients are

$$
A_{k p}=\sum_{m=0}^{p} \frac{c_{p m}}{s_{k}^{m}} \quad(p \leq k)
$$

and the notation

$$
\alpha(\varphi) \equiv \alpha_{k}\left(g_{k}(\varphi)\right)=1-e^{-2 \varphi}
$$

is used. All coefficients (37) are negative, e.g.

$$
\begin{gathered}
A_{11}=-\frac{1}{4}, \quad A_{21}=A_{11}, \quad A_{22}=-\frac{1}{12} \\
A_{31}=-0.388377, \quad A_{32}=-0.093205, \quad A_{33}=-0.016011 \\
A_{41}=A_{31}, \quad A_{42}=A_{32}, \quad A_{43}=A_{33}, \quad A_{44}=-0.003606 .
\end{gathered}
$$

The cascade velocity (16) is

$$
v_{k}(\varphi)=B_{k} \alpha^{k+1}(\varphi), \quad B_{k} \equiv A_{k+1, k+1} .
$$

Invoking the notation

$$
f_{k}^{*}(g) \equiv \ln \sqrt{1+z_{k}^{*}(g)}, \quad f_{k}(g) \equiv \ln \sqrt{1+z_{k}(g)}
$$

we can write the evolution integral (17) as

$$
\int_{z_{k}}^{z_{k+1}^{*}} \frac{(1+z)^{k}}{z^{k+1}} d z=2 B_{k} \tau
$$

where $z_{k}=z_{k}(g)$ and $z_{k}^{*}=z_{k}^{*}(g)$. Integrating Eq. (41), we obtain the equation

$$
z_{k+1}^{*}=z_{k} \exp \left\{P\left(\frac{1}{z_{k+1}^{*}}\right)-P\left(\frac{1}{z_{k}}\right)+2 B_{k} \tau\right\},
$$

defining $z_{k+1}^{*}(g)$, where

$$
P(x) \equiv \sum_{p=0}^{k-1} C_{k}^{p} \frac{x^{k-p}}{k-p}, \quad C_{k}^{p} \equiv \frac{k !}{(k-p) ! p !} .
$$


To check the stability of the approximation cascade $\left\{y_{k}\right\}$, we calculate the local multipliers (18). This gives

$$
\mu_{k}(\varphi)=1+2[1-\alpha(\varphi)] \sum_{p=1}^{k} p A_{k p} \alpha^{p-1}(\varphi) .
$$

It is interesting to note the relation between the subsequent multipliers,

$$
\mu_{k+1}(\varphi)=\mu_{k}(\varphi)+\Delta_{k}(\varphi)
$$

where

$$
\Delta_{k}(\varphi) \equiv 2(k+1) B_{k}[1-\alpha(\varphi)] \alpha^{k}(\varphi)
$$

From formulas (35) and (38), it follows that the domain of $g \in[0, \infty)$ corresponds to $\varphi \in[0, \infty)$ and $\alpha(\varphi) \in[0,1]$; that is, the effective expansion parameter $\alpha(\varphi)$ in Eqs. (36) and (43) is always less than 1 for arbitrary $g \in[0, \infty)$. Also, for all $\varphi \in[0, \infty)$ the stability conditions (19) and (21) are valid. Therefore, we put $\tau=1$ in Eq. (42).

It is convenient to characterize the accuracy of the approximants (40) by the corresponding maximal errors $\varepsilon_{k}^{*} \equiv \sup _{g}\left|\varepsilon_{k}^{*}(g)\right|, \varepsilon_{k} \equiv \sup _{g}\left|\varepsilon_{k}(g)\right|$. Comparing these approximants with direct numerical calculations for the function (26), we have $\varepsilon_{1}=7 \%$, $\varepsilon_{2}=$ $4 \%, \varepsilon_{3}=0.2 \%, \varepsilon_{4}=0.2 \% \ldots$, and $\varepsilon_{2}^{*}=3 \%, \varepsilon_{3}^{*}=2 \%, \varepsilon_{4}^{*}=0.1 \% \ldots$, which demonstrates good local convergence of the sequence $\left\{f_{k}^{*}(g)\right\}$ of the self-similar approximants $f_{k}^{*}(g)$.

\section{Anharmonic Oscillators}

In $0+1$ dimensions the $\varphi^{4}$ theory reduces to the familiar anharmonic oscillator problem. Therefore, it is instructive to illustrate the approach for the eigenvalue problem of quantum mechanics. Let us consider the Hamiltonian

$$
H(x)=-\frac{1}{2 m} \frac{d^{2}}{d x^{2}}+A x^{\nu},
$$

in which $m, A, \nu>0$, and $x \in \mathbf{R}$. This Hamiltonian, by scaling its variable, can be presented in the dimensionless form

$$
H=-\frac{1}{2} \frac{d^{2}}{d x^{2}}+g x^{\nu}
$$


To return from Eq. (47) to Eq. (46), one has to make the substitution

$$
H \rightarrow \frac{H(x)}{\omega}, \quad x \rightarrow \sqrt{m \omega} x, \quad g \rightarrow 1, \quad \omega^{2+\nu} \equiv \frac{A^{2}}{m^{\nu}} .
$$

So, we need to find the eigenvalues $e(n, g)$, where $n=0,1,2, \ldots$, of the Hamiltonian (47), and then, according to Eq. (48), we have to put $g=1$ obtaining the sought spectrum $e(n) \equiv e(n, 1)$. The latter can be compared with the direct numerical calculation of the Schrödinger equation, as well as with the quasiclassical approximation [52] which gives

$$
e_{W K B}(n)=\left[\sqrt{\frac{\pi}{2}}\left(n+\frac{1}{2}\right)\left(1+\frac{\nu}{2}\right) \Gamma\left(\frac{2+\nu}{2 \nu}\right) / \Gamma\left(\frac{1}{\nu}\right)\right]^{2 \nu /(2+\nu)},
$$

where $\Gamma(\cdot)$ is the Gamma-function.

For $\nu=2$, the Hamiltonian (47) is that of harmonic oscillator. This suggests to take for the initial approximation the harmonic oscillator

$$
H_{0}=-\frac{1}{2} \frac{d^{2}}{d x^{2}}+\frac{u^{2}}{2} x^{2}
$$

in which $u$ is a trial parameter that will generate later control functions $u_{k}(g)$. The eigenvalues and eigenfunctions of $H_{0}$ are, respectively,

$$
E_{0}(n, g, u)=\left(n+\frac{1}{2}\right) u, \quad \psi_{n}(x)=\frac{(u / \pi)^{1 / 4}}{\left(2^{n} n !\right)^{1 / 2}} \exp \left(-\frac{u}{2} x^{2}\right) H_{n}(\sqrt{u} x)
$$

where $n=0,1,2, \ldots$ and $H_{n}(\cdot)$ is a Hermit polynomial. By means of the RayleighSchrödinger perturbation theory, with respect to the perturbation $H-H_{0}$, one can find the sequence $\left\{E_{k}(n, g, u)\right\}$ of the $k$-order approximations for the eigenvalues of the Hamiltonian (47). For what follows, it is convenient to introduce the notation

$$
E_{k}(n, g, u) \equiv\left(n+\frac{1}{2}\right) F_{k}(n, g, u)
$$

and to consider the sequence $\left\{F_{k}(n, g, u)\right\}$, with $k=0,1,2, \ldots$ Defining control functions $u_{k}(n, g)$ from the fixed-point equation (15) and substituting them into Eq. (51), we get

$$
e_{k}(n)=\left(n+\frac{1}{2}\right) \lim _{g \rightarrow 1} F_{k}\left(n, g, u_{k}(n, g)\right) \text {. }
$$

And from the evolution integral (17), we find the self-similar approximant $e_{k}^{*}(n)$. 
Quartic oscillator. Let us realize the above program for $\nu=4$ in the Hamiltonian (46). For the perturbative terms $F_{k}$, defined in Eq. (51), limiting ourselves by the secondorder approximation, we have

$$
\begin{gathered}
F_{0}(n, g, u)=u, \quad F_{1}(n, g, u)=F_{0}(n, g, u)-\frac{u}{2}+\frac{3 g}{2 u^{2}} \gamma_{n}, \\
F_{2}(n, g, u)=F_{1}(n, g, u)-\frac{u}{8}+\frac{3 g}{2 u^{2}} \gamma_{n}-\frac{5 g^{2}}{2 u^{5}} \alpha_{n},
\end{gathered}
$$

where

$$
\gamma_{n} \equiv \frac{n^{2}+n+\frac{1}{2}}{n+\frac{1}{2}}=n+\frac{1}{2}+\frac{1}{4\left(n+\frac{1}{2}\right)}, \quad \alpha_{n} \equiv 1+\frac{27}{10}\left(n+\frac{1}{2}\right) \gamma_{n}-\left(n+\frac{1}{2}\right)^{2}
$$

For the control function we obtain $u_{1}(n, g)=\left(\begin{array}{lll}6 \gamma_{n} & g\end{array}\right)^{1 / 3}$. Equation (4) now reads $u_{k}\left(n, g_{k}(n, \varphi)\right)=\varphi$, which results in the coupling function $g_{1}(n, \varphi)=\varphi^{3} / 6 \gamma_{n}$. The endomorphism (5) is written as $y_{k}(n, \varphi)=\mu_{k}(n) \varphi$, where

$$
\mu_{1}(n)=\frac{3}{4}, \quad \mu_{2}(n)=\frac{3}{4}+\Delta(n), \quad \Delta(n) \equiv \frac{1}{8}-\frac{5 \alpha_{n}}{72 \gamma_{n}^{2}} .
$$

The cascade velocity (16) becomes $v_{1}(n, \varphi)=\Delta(n) \varphi$. Formula (52) gives

$$
e_{1}(n)=\frac{3}{4}\left(n+\frac{1}{2}\right)\left(6 \gamma_{n}\right)^{1 / 3}, \quad e_{2}(n)=\left[1+\frac{4}{3} \Delta(n)\right] e_{1}(n)
$$

And from the evolution integral (17), we obtain

$$
e_{2}^{*}(n)=e_{1}(n) \exp \{\Delta(n) \tau\}
$$

To decide what effective time $\tau$ to choose in the self-similar approximant (56), we have to analize the multipliers (18) and (20). For the local multipliers (18), we get $\mu_{k}(n, \varphi)=\mu_{k}(n)$, with $\mu_{k}(n)$ given in Eq. (54). When varying $n=0,1,2, \ldots \infty$, we have

$$
\frac{35}{48} \leq \mu_{2}(n) \leq \frac{109}{144}, \quad-\frac{1}{48} \leq \Delta(n) \leq \frac{1}{144}
$$

Defining the maximal multipliers

$$
\mu_{k} \equiv \sup _{n}\left|\mu_{k}(n)\right|, \quad \bar{\mu}_{k} \equiv \sup _{n}\left|\bar{\mu}_{k}(n)\right|,
$$


we obtain $\mu_{1}=0.75, \mu_{2}=0.756944, \bar{\mu}_{2}=1.009259$. It is the maximal multipliers (57) that are to be considered in the stability analysis. This is because the sequence $\left\{F_{k}\right\}$, as in Eq. (53), has been derived employing the Raleigh-Schrödinger perturbation theory, which involves the summation over quantum numbers. Therefore, the terms $F_{k}(n, g, u)$, and consequently the energies (51) and (52), with different quantum numbers $n$, cannot be treated as completely independent.

Since $\mu_{k}<1$, the procedure is locally stable; but $\bar{\mu}_{2}$ is slightly larger than 1 , so that there is a week ultralocal instability. Thus, we need to take the middle point $\tau=\frac{1}{2}$; although, because the ultralocal instability is so weak, we could expect that the results should not essentially differ between the cases $\tau=\frac{1}{2}$ and $\tau=1$.

To check the prediction of the stability analysis, we may compare the energy levels (55) and (56) with the results of numerical solution of the corresponding Schrödinger equation [53]. Varying the quantum numbers $n=0,1,2, \ldots$, we define the maximal errors

$$
\varepsilon_{W K B} \equiv \sup _{n}\left|\varepsilon_{W K B}(n)\right|, \quad \varepsilon_{k} \equiv \sup _{n}\left|\varepsilon_{k}(n)\right|, \quad \varepsilon_{k}^{*} \equiv \sup _{n}\left|\varepsilon_{k}^{*}(n)\right| .
$$

For the latter we find $\varepsilon_{W K B}=1.5 \%, \varepsilon_{1}=2 \%, \varepsilon_{2}=0.8 \%, \varepsilon_{2}^{*}=0.4 \%$. The error $\varepsilon_{2}^{*}$ does not change for the alternative choices of $\tau=1$ and $\tau=\frac{1}{2}$.

Sextic oscillator. For the power $\nu=6$ in the Hamiltonian (46), we have the sequence

$$
\begin{gathered}
F_{0}(n, g, u)=u, \quad F_{1}(n, g, u)=F_{0}(n, g, u)-\frac{u}{2}+\frac{5 g}{2 u^{3}} \kappa_{n}, \\
F_{2}(n, g, u)=F_{1}(n, g, u)-\frac{u}{8}+\frac{15 g}{4 u^{3}} \kappa_{n}-\frac{g^{2}}{32 u^{7}} \beta_{n},
\end{gathered}
$$

which is defined in Eq. (51), and where

$$
\kappa_{n} \equiv n^{2}+n+\frac{3}{2}, \quad \beta_{n} \equiv 786 n^{4}+1572 n^{3}+5324 n^{2}+4538 n+3495 .
$$

Then we follow the same steps as for the quartic oscillator. We find the control function $u_{1}(n, g)=\left(15 \kappa_{n} g\right)^{1 / 4}$ and the coupling function $g_{1}(n, \varphi)=\varphi^{4} / 15 \kappa_{n}$. Constructing the approximation cascade, we come to the same endomorphism $y_{k}(n, \varphi)=\mu_{k}(n) \varphi$ but with

$$
\mu_{1}(n)=\frac{2}{3}, \quad \mu_{2}=\frac{2}{3}+\Delta(n), \quad \Delta(n) \equiv \frac{1}{8}-\frac{\beta_{n}}{7200 \kappa_{n}^{2}} .
$$


From Eq. (52), we get

$$
e_{1}(n)=\frac{2}{3}\left(n+\frac{1}{2}\right)\left(15 \kappa_{n}\right)^{1 / 4}, \quad e_{2}(n)=\left[1+\frac{3}{2} \Delta(n)\right] e_{1}(n) .
$$

The integral (17) gives the same expression (56), but with $\Delta(n)$ defined in Eq. (60).

For the local multipliers (18), we have the same form as in the previous case, with $\mu_{k}(n)$ given in Eq. (60). Varying the quantum numbers $n=0,1,2, \ldots$, we find

$$
\frac{311}{540} \leq \mu_{2}(n) \leq \frac{273}{400}, \quad-\frac{19}{1200} \leq \Delta(n) \leq \frac{49}{540} .
$$

The maximal multipliers (57) are $\mu_{1}=0.6667, \mu_{2}=0.6825, \bar{\mu}_{2}=1.023699$. Again we see that the procedure is locally stable since $\mu_{k}<1$, but with a weak ultralocal instability because of $\bar{\mu}_{2}>1$. Therefore, we again have to take in Eq. (58) the middle point $\tau=\frac{1}{2}$. For the maximal errors (58), with respect to numerical calculations [54], we find $\varepsilon_{W K B}=4 \%, \varepsilon_{1}=7 \%, \varepsilon_{2}=8 \%, \varepsilon_{2}^{*}=2 \%$. Taking $\tau=1$ slightly increase the error to $3 \%$. Note that in all cases the maximal error occurs at the ground-state level.

Octic oscillator. With the power $\nu=8$ in the Hamiltonian (46), we find the sequence of perturbative terms

$$
\begin{gathered}
F_{0}(n, g, u)=u, \quad F_{1}(n, g, u)=F_{0}(n, g, u)-\frac{u}{2}+\frac{35 g}{8 u^{4}} \sigma_{n}, \\
F_{2}(n, g, u)=F_{1}(n, g, u)-\frac{u}{8}+\frac{35 g}{4 u^{4}} \sigma_{n}-\frac{g^{2}}{32 u^{9}} \delta_{n},
\end{gathered}
$$

where

$$
\begin{gathered}
\sigma_{n} \equiv \frac{n^{4}+2 n^{3}+5 n^{2}+4 n+3 / 2}{n+1 / 2} \\
\delta_{n} \equiv 3985 n^{6}+11955 n^{5}+74904 n^{4}+129883 n^{3}+277901 n^{2}+214952 n+135030 .
\end{gathered}
$$

The control function is $u_{1}(n, g)=\left(35 \sigma_{n} g\right)^{1 / 5}$ and the coupling function becomes $g_{1}(n, \varphi)=$ $\varphi^{5} / 35 \sigma_{n}$. The approximation cascade is described by the same endomorphism with

$$
\mu_{1}(n)=\frac{5}{8}, \quad \mu_{2}=\frac{5}{8}+\Delta(n), \quad \Delta(n) \equiv \frac{1}{8}-\frac{\delta_{n}}{39200 \sigma_{n}^{2}} .
$$

For varying $n=0,1,2, \ldots$, one has

$$
\frac{4319}{11760} \leq \mu_{2}(n) \leq \frac{5083}{7840}, \quad-\frac{3031}{11760} \leq \Delta(n) \leq \frac{183}{7840} .
$$


The energies (52) are

$$
e_{1}(n)=\frac{5}{8}\left(n+\frac{1}{2}\right)\left(35 \sigma_{n}\right)^{1 / 5}, \quad e_{2}(n)=\left[1+\frac{8}{5} \Delta(n)\right] e_{1}(n) .
$$

And the self-similar approximant $e_{2}^{*}(n)$ again has the same form (56).

The local multipliers are given by $\mu_{k}(n)$. For the maximal multipliers (57) we find $\mu_{1}=0.625, \mu_{2}=0.648342, \bar{\mu}_{2}=1.037347$. This shows that the procedure is locally stable but is not ultralocally stable. So, the self-similar approximant (56) has to be defined at the middle point $\tau=\frac{1}{2}$.

For the maximal errors (58), with respect to numerical calculations [54], we obtain $\varepsilon_{W K B}=7 \%, \varepsilon_{1}=13 \%, \varepsilon_{2}=34 \%, \varepsilon_{2}^{*}=3 \%$. The accuracy of the self-similar approximant (56) for $\tau=\frac{1}{2}$ is essentially higher than that for $\tau=1$, for which case the error reaches $13 \%$.

Multidimensional oscillator. In the previous three cases we have shown that the method works well for different one-dimensional anharmonic oscillators. Now we want to demonstrate that it is equally applicable for anharmonic oscillators of arbitrary dimensionality. For this purpose, we consider the spherically symmetric quartic oscillator in $D$-dimensional real space. The corresponding radial Hamiltonian reads

$$
H(r)=-\frac{1}{2 m} \frac{d^{2}}{d r^{2}}+\frac{(2 l+D-3)(2 l+D-1)}{8 m r^{2}}+A r^{4}
$$

where $m, A>0 ; l=0,1,2, \ldots ; r \geq 0$, and $D=1,2,3, \ldots$ is a real-space dimensionality. This Hamiltonian, by the appropriate scaling, can be reduced to the form

$$
H=-\frac{1}{2} \frac{d^{2}}{d r^{2}}+\frac{(2 l+D-3)(2 l+D-1)}{8 r^{2}}+g r^{4} .
$$

One can return from Eq. (66) back to Eq. (65) by means of the substitution

$$
H \rightarrow \frac{H(r)}{\omega}, \quad r \rightarrow \sqrt{m \omega} r, \quad g \rightarrow 1, \quad \omega \equiv\left(\frac{A}{m^{2}}\right)^{1 / 3}
$$

We start perturbation theory with the harmonic $D$-dimensional oscillator defined by the Hamiltonian

$$
H_{0}=-\frac{1}{2} \frac{d^{2}}{d r^{2}}+\frac{(2 l+D-3)(2 l+D-1)}{8 r^{2}}+\frac{u^{2}}{2} r^{2},
$$


in which $u$ is a trial parameter. The corresponding eigenvalues are

$$
E_{0}(n, l, u)=\left(2 n+l+\frac{D}{2}\right) u
$$

where $n=0,1,2, \ldots$, and the eigenfunctions are

$$
\chi_{n l}(r)=\left[\frac{2 n ! u^{l+D / 2}}{\Gamma(n+l+D / 2)}\right]^{1 / 2} r^{(2 l+D-1) / 2} \exp \left(-\frac{u}{2} r^{2}\right) L_{n}^{(2 l+D-2) / 2}\left(u r^{2}\right)
$$

where $L_{n}^{l}(r)$ is the associate Laguerre polynomial. The latter can be presented in several forms, so we write down below that one we use in what follows:

$$
L_{n}^{l}(r)=\frac{1}{n !} e^{r} r^{-l} \frac{d^{n}}{d r^{n}}\left(e^{-r} r^{n+l}\right)=\sum_{m=0}^{n} \frac{\Gamma(n+l+1)(-r)^{m}}{\Gamma(m+l+1) m !(n-m) !} .
$$

For the terms of perturbation theory, we use the notation

$$
E_{k}(n, l, g, u) \equiv\left(2 n+l+\frac{D}{2}\right) F_{k}(n, l, g, u) .
$$

Then, in the second order we have

$$
\begin{gathered}
F_{0}(n, l, g, u)=u, \quad F_{1}(n, l, g, u)=F_{0}(n, l, g, u)-\frac{u}{2}+\frac{3 g}{2 u^{2}} \gamma_{n l} \\
F_{2}(n, l, g, u)=F_{1}(n, l, g, u)-\frac{u}{8}+\frac{3 g}{2 u^{2}} \gamma_{n l}-\frac{5 g^{2}}{2 u^{5}} \alpha_{n l}
\end{gathered}
$$

where

$$
\begin{gathered}
\gamma_{n l} \equiv 2 n+l+\frac{D}{2}-\frac{(l+D / 2)(l-2+D / 2)}{3(2 n+l+D / 2)}, \\
\alpha_{n l} \equiv 1+\frac{27}{10}\left(2 n+l+\frac{D}{2}\right) \gamma_{n l}-\left(2 n+l+\frac{D}{2}\right)^{2} .
\end{gathered}
$$

The one-dimensional case can be recovered with the change $D \rightarrow 1,2 n \rightarrow n, l \rightarrow 0$.

All steps of the procedure are the same as earlier, so we do not go into much details. For the control functions we find $u_{1}(n, l, g)=\left(6 \gamma_{n l} g\right)^{1 / 3}$, and for the coupling function we get $g_{1}(n, l, \varphi)=\varphi^{3} / 6 \gamma_{n l}$.

The approximation cascade is characterized by the endomorphism $y_{k}(n, l, \varphi)=\mu_{k}(n, l) \varphi$, in which

$$
\mu_{1}(n, l)=\frac{3}{4}, \quad \mu_{2}(n, l)=\frac{3}{4}+\Delta(n, l), \quad \Delta(n, l) \equiv \frac{1}{8}-\frac{5 \alpha_{n l}}{72 \gamma_{n l}^{2}}
$$


and the cascade velocity is $v_{1}(n, l, \varphi)=\Delta(n, l) \varphi$.

Similarly to Eq. (52), we define

$$
e_{k}(n, l)=\left(2 n+l+\frac{D}{2}\right) \lim _{g \rightarrow 1} F_{k}\left(n, l, g, u_{k}(n, l, g)\right) .
$$

This yields

$$
e_{1}(n, l)=\frac{3}{4}\left(2 n+l+\frac{D}{2}\right)\left(6 \gamma_{n l}\right)^{1 / 3}, \quad e_{2}(n, l)=\left[1+\frac{4}{3} \Delta(n, l)\right] e_{1}(n, l) .
$$

And the evolution integral (17) gives us the self-similar approximant

$$
e_{2}^{*}(n, l)=e_{1}(n, l) \exp \{\Delta(n, l) \tau\}
$$

The stability analysis here is practically the same as for the one-dimensional quartic oscillator, with the same conclusion that the procedure is locally stable but with a very weak ultralocal instability. Consequently, in the approximant (70) one has to take the middle point $\tau=\frac{1}{2}$, although the results should not be much different from the case $\tau=1$. The accuracy should increase with the increasing dimensionality $D$, since increasing $D$

supresses ultralocal instability. For example, when $D \rightarrow \infty$, then $\gamma_{n l} \rightarrow D / 3, \alpha_{n l} \rightarrow D^{2} / 5$, and $\Delta(n, l) \rightarrow 0$, so that $\bar{\mu}_{2} \rightarrow 1$. These conclusions are in perfect agreement with calculations. Thus, the maximal error of the self-similar approximant (70) is $\varepsilon_{2}^{*}=0.4 \%$ for $D=1$ and it is $\varepsilon_{2}^{*}=0.3 \%$ for $D=3$.

\section{$5 \quad$ Arbitrary Powers}

In the previous section, we considered several Hamiltonians with potentials having even integer powers. However, this restriction is not principal, and the method can be applied to the case of potentials with arbitrary, noninteger as well as integer, powers. To demonstrate this, let us consider a spherically symmetric three-dimensional problem with the radial Hamiltonian

$$
H(r)=-\frac{1}{2 m} \frac{d^{2}}{d r^{2}}+\frac{l(l+1)}{2 m r^{2}}+A r^{\nu},
$$


in which $m, A>0 ; l=0,1,2, \ldots ; r \geq 0$; and $\nu>0$ is an arbitrary positive number. By scaling, it is again convenient to reduce this Hamiltonian to the simplified form

$$
H=-\frac{1}{2} \frac{d^{2}}{d r^{2}}+\frac{l(l+1)}{2 r^{2}}+g r^{\nu}
$$

The return from Eq. (72) to (71) is made by means of the substitution

$$
H \rightarrow \frac{H(r)}{\omega}, \quad r \rightarrow \sqrt{m \omega} r, \quad g \rightarrow 1, \quad \omega^{2+\nu} \equiv \frac{A^{2}}{m^{\nu}} .
$$

Hamiltonians of this type have been considered in a number of papers (see e.g. [31,55-58] and references therein). The importance of potentials with various powers is due to the fact that such potentials model well confining forces between heavy quarks and lead to a reasonable description of the quarkonium spectrum [59]. The quasiclassical approximation gives [59] the eigenvalues of the Hamiltonian (72) in the form

$$
e_{W K B}(n, l)=\left[\sqrt{\frac{\pi}{2}}\left(2 n+l+\frac{3}{2}\right)\left(1+\frac{\nu}{2}\right) \Gamma\left(\frac{2+\nu}{2 \nu}\right) / \Gamma\left(\frac{1}{\nu}\right)\right]^{2 \nu /(2+\nu)} .
$$

Applying our method to the eigenvalue problem for the Hamiltonian (72), we start with the harmonic Hamiltonian

$$
H_{0}=-\frac{1}{2} \frac{d^{2}}{d r^{2}}+\frac{l(l+1)}{2 r^{2}}+\frac{u^{2}}{2} r^{2}
$$

whose eigenvalues are

$$
E_{0}(n, l, u)=\left(2 n+l+\frac{3}{2}\right) u .
$$

Using the Rayleigh-Schrödinger perturbation theory, we can find the $k$-order terms

$$
E_{k}(n, l, g, u) \equiv\left(2 n+l+\frac{3}{2}\right) F_{k}(n, l, g, u)
$$

Then, defining control functions $u_{k}(n, l, g)$, we come to the renormalized eigenvalues

$$
e_{k}(n, l) \equiv\left(2 n+l+\frac{3}{2}\right) \lim _{g \rightarrow 1} F_{k}\left(n, l, g, u_{k}(n, l, g)\right)
$$

In the second order, we obtain the sequence

$$
F_{0}(n, l, g, u)=u, \quad F_{1}(n, l, g, u)=F_{0}(n, l, g, u)-\frac{u}{2}+\frac{g}{u^{\nu / 2}} A_{n l}\left(\frac{\nu}{2}\right)
$$




$$
F_{2}(n, l, g, u)=F_{1}(n, l, g, u)-\frac{u}{8}+\frac{g}{2 u^{\nu / 2}} B_{n l}\left(\frac{\nu}{2}\right)-\frac{g^{2}}{2 u^{1+\nu}} C_{n l}\left(\frac{\nu}{2}\right),
$$

with the coefficients

$$
\begin{gathered}
A_{n l}(\nu) \equiv \frac{n ! I_{n n}^{l}(\nu)}{(2 n+l+3 / 2) \Gamma(n+l+3 / 2)}, \\
B_{n l}(\nu) \equiv \frac{n !}{(2 n+l+3 / 2) \Gamma(n+l+3 / 2)}\left[(n+1) I_{n, n+1}^{l}(\nu)-\left(n+l+\frac{1}{2}\right) I_{n, n-1}^{l}(\nu)\right] \\
C_{n l}(\nu) \equiv \frac{n !}{(2 n+l+3 / 2) \Gamma(n+l+3 / 2)} \sum_{p=0(p \neq n)}^{\infty} \frac{p !\left[I_{n p}^{l}(\nu)\right]^{2}}{(p-n) \Gamma(p+l+3 / 2)} .
\end{gathered}
$$

Here we use the notation

$$
I_{n s}^{l}(\nu) \equiv \int_{0}^{\infty} t^{l+\nu+1 / 2} e^{-t} L_{n}^{l+1 / 2}(t) L_{s}^{l+1 / 2}(t) d t
$$

where $L_{n}^{l}(t)$ is an associate Laguerre polynomial. The way of calculating this integral is explained in Appendix A. As is seen from the sequence (76), we have there an expression in powers of $g / u^{1+\nu / 2}$.

Following the same steps as in the previous section, we find the control and coupling functions

$$
u_{1}(n, l, g)=\left[\nu A_{n l}\left(\frac{\nu}{2}\right) g\right]^{2 /(2+\nu)}, \quad g_{1}(n, l, \varphi)=\frac{\varphi^{1+\nu / 2}}{\nu A_{n l}(\nu / 2)}
$$

The approximation cascade is defined by the endomorphism $y_{k}(n, l, \varphi)=\mu_{k}(n, l) \varphi$ with

$$
\begin{gathered}
\mu_{1}(n, l)=\frac{2+\nu}{2 \nu}, \quad \mu_{2}(n, l)=\frac{2+\nu}{2 \nu}+\Delta(n, l), \\
\Delta(n, l) \equiv \frac{B_{n l}(\nu / 2)}{2 \nu A_{n l}(\nu / 2)}-\frac{C_{n l}(\nu / 2)}{2 \nu^{2} A_{n l}(\nu / 2)}-\frac{1}{8} .
\end{gathered}
$$

For the values (75), we have

$$
\begin{gathered}
e_{1}(n, l)=\left(2 n+l+\frac{3}{2}\right) \frac{2+\nu}{2 \nu}\left[\nu A_{n l}\left(\frac{\nu}{2}\right)\right]^{2 /(2+\nu)}, \\
e_{2}(n, l)=\left[1+\frac{2 \nu}{2+\nu} \Delta(n, l)\right] e_{1}(n, l) .
\end{gathered}
$$

The evolution integral (17) yields the self-similar approximant

$$
e_{2}^{*}(n, l)=e_{1}(n, l) \exp \{\Delta(n, l) \tau\}
$$


The local multipliers $(18)$ are $\mu_{k}(n, l, \varphi)=\mu_{k}(n, l)$, with the right-hand side defined in Eq. (78). The analysis of these multipliers shows that the stability properties depend on the power $\nu$ of the potential in the Hamiltonian (72). For $\nu \neq 2$, the procedure is either locally unstable but then it is ultralocally stable, or the procedure is locally stable but ultralocally unstable. In both these cases, we have to take in the approximant (80) the middle point $\tau=\frac{1}{2}$. The case $\nu=2$ is special. Then all multipliers $\mu_{k}=\bar{\mu}_{k}=1$, which corresponds to the neutral stability. This means that we are already at a fixed point of the cascade trajectory, and there is no motion any more. Really, our calculations show that for $\nu=2$ and any $\tau$, we have for all eigenvalues $e_{1}(n, l)=e_{2}(n, l)=e_{2}^{*}(n, l)$, which coincides with the exact solution for the harmonic oscillator. To illustrate what are the values of typical errors characterizing the accuracy of different approximations, we present in Table I the results of calculations of the ground-state energy for various powers $\nu$. We give there the errors $\varepsilon_{1}$ and $\varepsilon_{2}$ for the renormalized expressions from Eq. (79), the error $\varepsilon_{2}^{*}$ for the self-similar approximant (80), and also the error of the quasiclassical approximation (73). These errors are computed by comparing the results of our calculations with accurate numerical data $[55,57,59]$ obtained by the direct numerical solution of the Schrödinger equation. The results for the approximant (80) correspond to the middle-point case $\tau=\frac{1}{2}$, which is taken according to the stability analysis. For comparison, we also made calculations setting $\tau=1$. The latter case leads to the errors that are close to $\varepsilon_{2}^{*}$ from Table I, when $\nu \leq 6$. However, for $\nu>6$, the errors quickly increase becoming, e.g., $-6 \%$ for $\nu=8$ and $-19 \%$ for $\nu=10$. Hence, the middle point choice $\tau=\frac{1}{2}$ is especially preferable for large powers of the potential.

\section{Initial Approximations}

Now we aim at analysing the following problem. Assume that we can take not just one but two or more initial approximations that can be employed as starting points for constructing the corresponding approximation cascades. Then how to decide which of these initial approximations is preferable? We show that the stability analysis again can 
give us an answer to this question.

For the purpose under consideration, it is convenient to take the logarithmic potential whose behaviour is such that it can be modelled, to some extent, either by the harmonic oscillator or by the Coulomb potential. We mean here the three-dimensional spherically symmetric problem with the radial Hamiltonian

$$
H(r)=-\frac{1}{2 m} \frac{d^{2}}{d r^{2}}+\frac{l(l+1)}{2 m r^{2}}+B \ln \frac{r}{b},
$$

in which $m, B, b>0$, and $r \geq 0$. As usual, we transform the Hamiltonian (81) to the scaled form

$$
H=-\frac{1}{2} \frac{d^{2}}{d r^{2}}+\frac{l(l+1)}{2 r^{2}}+g \ln r .
$$

The return from Eq. (82) to Eq. (81) is made by means of the substitution

$$
H \rightarrow \frac{H(r)}{\omega}, \quad r \rightarrow \frac{r}{b}, \quad g \rightarrow \frac{B}{\omega}, \quad \omega \equiv \frac{1}{m b^{2}} .
$$

Note that the logarithmic potential is also often used for describing confining forces between quarks [59], and the quasiclassical approximation for the spectrum of the Hamiltonian (82) is known to be

$$
e_{W K B}(n, l, g)=\frac{g}{2} \ln \left[\frac{\pi}{2 g}\left(2 n+l+\frac{3}{2}\right)^{2}\right] .
$$

Harmonic potential. Let us choose for the initial approximation the harmonicoscillator Hamiltonian. Then for the function

$$
F_{k}(n, l, g, u) \equiv \frac{E_{k}(n, l, g, u)}{(2 n+l+3 / 2)}
$$

we derive the sequence of perturbative terms

$$
\begin{gathered}
F_{0}(n, l, g, u)=u \\
F_{1}(n, l, g, u)=F_{0}(n, l, g, u)-\frac{u}{2}+g \frac{\psi(n+l+3 / 2)-\ln u}{2(2 n+l+3 / 2)}, \\
F_{2}(n, l, g, u)=F_{1}(n, l, g, u)-\frac{u}{8}+\frac{g}{4(2 n+l+3 / 2)}-\frac{g^{2}}{8 u} C_{n l},
\end{gathered}
$$


where

$$
\begin{gathered}
\psi(x) \equiv \frac{d}{d x} \ln \Gamma(x), \\
C_{n l} \equiv \frac{n !}{(2 n+l+3 / 2) \Gamma(n+l+3 / 2)} \sum_{p=0(p \neq n)}^{\infty} \frac{p !\left(I_{n p}^{l}\right)^{2}}{(p-n) \Gamma(p+l+3 / 2)},
\end{gathered}
$$

Here $I_{n s}^{l}$ denotes the integral

$$
I_{n s}^{l} \equiv \int_{0}^{\infty} t^{l+1 / 2} e^{-t} \ln t L_{n}^{l+1 / 2}(t) L_{s}^{l+1 / 2}(t) d t
$$

The properties of this integral are described in Appendix B.

Following the standard scheme of our method, we find the control and coupling functions

$$
u_{1}(n, l, g)=\frac{g}{2 n+l+3 / 2}, \quad g_{1}(n, l, \varphi)=\left(2 n+l+\frac{3}{2}\right) \varphi .
$$

Then we construct the approximation cascade $\left\{y_{k}\right\}$, with the trajectory points

$$
y_{1}(n, l, \varphi)=\frac{\varphi}{2}\left[1-\ln \varphi+\psi\left(n+l+\frac{3}{2}\right)\right], \quad y_{2}(n, l, \varphi)=y_{1}(n, l, \varphi)+v_{1}(n, l, \varphi)
$$

and with the cascade velocity $v_{1}(n, l, \varphi)=\Delta(n, l) \varphi$, where

$$
\Delta(n, l) \equiv \frac{1}{8}-\frac{1}{8}\left(2 n+l+\frac{3}{2}\right)^{2} C_{n l}, \quad \Delta(0,0)=-0.018288 .
$$

For the renormalized expressions

$$
e_{k}(n, l, g)=\left(2 n+l+\frac{3}{2}\right) F_{k}\left(n, l, g, u_{k}(n, l, g)\right)
$$

we have

$$
\begin{gathered}
e_{1}(n, l, g)=\frac{g}{2}\left[1+\ln \frac{2 n+l+3 / 2}{g}+\psi\left(n+l+\frac{3}{2}\right)\right], \\
e_{2}(n, l, g)=e_{1}(n, l, g)+\Delta(n, l) g .
\end{gathered}
$$

From the evolution integral (17), we obtain the self-similar approximant

$$
e_{2}^{*}(n, l, g)=e_{1}(n, l, g) \exp \{\Delta(n, l) \tau\}
$$


The qualitative behaviour of the energy (90) is as follows. At small $g \rightarrow+0$, this energy is positive and tends to zero as $-g \ln g$. As $g$ increases, $e_{2}^{*}$ increases till the maximum value

$$
e_{2}^{*}\left(n, l, g_{\max }\right)=\frac{1}{2} g_{\max }(n, l) \exp \{\Delta(n, l) \tau\}
$$

occurring at

$$
g_{\text {max }}(n, l)=\left(2 n+l+\frac{3}{2}\right) \exp \left\{\psi\left(n+l+\frac{3}{2}\right)\right\} .
$$

With the further increase of $g$, function (90) decreases to zero, $e_{2}^{*}\left(n, l, g_{0}\right)=0$, at the value

$$
g_{0}(n, l)=e g_{\max }(n, l)
$$

after which $e_{2}^{*}$ remains negative. Using notation (92), we may present Eq. (90) as

$$
e_{2}^{*}(n, l, g)=\frac{g}{2} \ln \left(\frac{g_{0}}{g}\right) \exp \{\Delta(n, l) \tau\},
$$

where $g_{0}=g_{0}(n, l)$.

To estimate the values of the characteristic couplings (91) and (92), consider the case $\mathrm{n}=\mathrm{l}=0$, when

$$
g_{\max }(0,0)=\frac{3}{8} e^{2-C}=1.555746, \quad g_{0}(0,0)=\frac{3}{8} e^{3-C}=4.228956
$$

where $C$ is the Euler constant.

In the opposite case, when either $n$ or $l$ is large, since $\psi(x) \simeq \ln x$, as $x \rightarrow \infty$, we have

$$
g_{\max }(n, l) \simeq\left\{\begin{array}{cc}
2 n^{2} & (n \rightarrow \infty, l<\infty) \\
l^{2} & (n<\infty, l \rightarrow \infty)
\end{array}\right.
$$

The local multipliers (18), for the trajectories points (87), are

$$
\mu_{1}(n, l, \varphi)=\frac{1}{2} \psi\left(n+l+\frac{3}{2}\right)-\frac{1}{2} \ln \varphi, \quad \mu_{2}(n, l, \varphi)=\mu_{1}(n, l, \varphi)+\Delta(n, l) .
$$

The images of the multipliers (96), defined in Eq. (22), become

$$
\begin{gathered}
m_{1}(n, l, g)=\frac{1}{2} \psi\left(n+l+\frac{3}{2}\right)+\frac{1}{2} \ln \frac{2 n+l+3 / 2}{g} \\
m_{2}(n, l, g)=m_{1}(n, l, g)+\Delta(n, l)
\end{gathered}
$$


With definition (91), we may write

$$
m_{1}(n, l, g)=\frac{1}{2} \ln \frac{g_{\max }(n, l)}{g} .
$$

Varying the quantum numbers $n$ and $l$, one can check that $\Delta(n, l)$ is always negative and the ultralocal multiplier $\bar{m}_{2}(n, l, g) \equiv m_{2}(n, l, g) / m_{1}(n, l, g)$, which is the image of the multiplier (20), is less than one for all $n, l$, and $g$. That is, the procedure is ultralocally stable. However, it is locally stable not for all coupling parameters but only for those satisfying the inequalities

$$
\frac{1}{e^{2}}<\frac{g}{g_{\max }}<e^{2}
$$

where $g_{\max }=g_{\max }(n, l)$ is given by Eq. (91). For instance, if $n=l=0$, then the interval (99) is

$$
0.210547<g<11.495494
$$

In the region of stability (100), we may put $\tau=1$. For illustration, we present in Table II the results of calculations for $n=0$ and $l=0,1,2,3,4$, and for the coupling parameter $g=0.5$. We show the percentage errors $\varepsilon_{1}$ and $\varepsilon_{2}$ for the renormalized expressions (89), the error $\varepsilon_{2}^{*}$ of the self-similar approximant (90), and the error $\varepsilon_{W K B}$ of the quasiclassical approximation (83). The comparison is made with respect to numerical data $[55,59]$.

Coulomb potential. Now let us turn to the case when the Hamiltonian

$$
H_{0}=-\frac{1}{2} \frac{d^{2}}{d r^{2}}+\frac{l(l+1)}{2 r^{2}}+\frac{u}{r}
$$

with the Coulomb potential is chosen for the initial approximation. The corresponding eigenvalues and eigenfunctions are

$$
\begin{gathered}
E_{0}(n, l, g, u)=-\frac{u^{2}}{2(n+l+1)^{2}}, \\
\chi_{n l}(r)=\left[\frac{n ! u}{(n+2 l+1) !}\right]^{1 / 2} \frac{1}{n+l+1}\left(\frac{2 u r}{n+l+1}\right)^{l+1} \exp \left(-\frac{u r}{n+l+1}\right) L_{n}^{2 l+1}\left(\frac{2 u r}{n+l+1}\right) .
\end{gathered}
$$

In calculating matrix elements, we meet the integral

$$
J_{n s}^{l} \equiv \int_{0}^{\infty} t^{2 l+2} e^{-t} \ln t L_{n}^{2 l+1}(t) L_{s}^{2 l+1}(t) d t
$$


whose properties are specified in Appendix C. The whole calculational procedure is very similar to the previous subsection, because of which we shorten here technical details. This is especially justified by the fact that the procedure starting with the initial approximation corresponding to the Hamiltonian (101), as will be shown, is less stable than that beginning with the harmonic-oscillator Hamiltonian.

The spectrum in the first approximation reads

$$
E_{1}(n, l, g, u)=\frac{u^{2}}{2(n+l+1)^{2}}+g\left(\ln \frac{n+l+1}{2 u}+D_{n l}\right),
$$

where

$$
D_{n l} \equiv \frac{2 n+1}{2(n+l+1)}+\psi(n+2 l+2) .
$$

For the control and coupling functions, we find

$$
u_{1}(n, l, g) \equiv(n+l+1) \sqrt{g}, \quad g_{1}(\varphi)=-2 \varphi .
$$

Then the point of the approximation cascade, bijective to the approximation (103), is

$$
y_{1}(n, l, \varphi)=-\varphi\left[1-\ln (-8 \varphi)+2 D_{n l}\right] .
$$

Substituting the control function into spectrum (103) gives

$$
e_{1}(n, l, g)=\frac{g}{2}\left(1+\ln \frac{1}{4 g}+2 D_{n l}\right) .
$$

The self-similar approximant $e_{2}^{*}$ acquires the form of Eq. (90).

The overall qualitative behaviour of the spectrum is the same as in the previous subsection. For $g \rightarrow 0$, the energy tends to zero as $-g \ln g$. With increasing $g$, the energy reaches a maximum at the point

$$
g_{\text {max }}(n, l)=\frac{1}{4} \exp \left(2 D_{n l}\right) .
$$

Then the energy diminishes to zero at the value $g_{0}=g_{0}(n, l)$ having the same relation $g_{0}=e g_{\max }$, as in Eq. (92). For the ground-state level, we now have

$$
g_{\max }(0,0)=\frac{1}{4} e^{3-2 C}=1.582925, \quad g_{0}(0,0)=\frac{1}{4} e^{4-2 C}=4.302836 .
$$


The energy (104) can be written as

$$
e_{1}(n, l, g)=\frac{g}{2} \ln \left(\frac{g_{0}}{g}\right)
$$

where $g_{0}=g_{0}(n, l)$, and $e_{2}^{*}$ can be presented as in Eq. (93).

The characteristic values of $g_{\max }$, given by Eqs. (105) and (91), are very close to each other. This is seen from Eqs. (94) and (106), as well as comparing Eq. (95) with the corresponding behaviour of Eq. (105) yielding

$$
g_{\max }(n, l) \simeq\left\{\begin{array}{cc}
\left(e^{2} / 4\right) n^{2} & (n \rightarrow \infty, l<\infty) \\
l^{2} & (n<\infty, l \rightarrow \infty) .
\end{array}\right.
$$

For the local multiplier (18) and its image (22), we find

$$
\mu_{1}(n, l, \varphi)=\ln (-8 \varphi)-2 D_{n l}, \quad m_{1}(n, l, g)=\ln \frac{g}{g_{\max }(n, l)} .
$$

The stability condition $\left|\mu_{1}(n, l, \varphi)\right|<1,\left|m_{1}(n, l, \varphi)\right|<1$ is satisfied in the region

$$
\frac{1}{e}<\frac{g}{g_{\max }}<e,
$$

where $g_{\max }=g_{\max }(n, l)$ is defined in Eq. (105). For the ground state, the inequalities (109) give

$$
0.582326<g<4.302836 \text {. }
$$

Comparing the stability properties of the calculational procedure for the considered cases, when either the harmonic oscillator or the Coulomb potential are used for the initial approximation, we come to the following conclusion. The region of stability, with respect to the coupling parameter $g$, is three times narrower in the latter case than in the former. Also, the values of the local multipliers in the latter case are about twice as large as for the former case. Consequently, the calculational procedure starting with the harmonic oscillator is more stable than that beginning with the Coulomb potential. This conclusion is in agreement with calculations showing that the first, more stable, case is also more accurate than the second, less stable; the error of the latter case being almost twice larger. 


\section{Conclusion}

The self-similar perturbation theory makes it possible to obtain quite accurate approximations for those complicated physical problems for which only a few terms of a perturbative algorithm are available. The term "perturbation theory" here has to be understood in the general mathematical sense as a regular procedure yielding a set of subsequent approximations. This approach does not require any small parameters of the physical problem considered. For instance, the coupling parameters can be arbitrary strong. Convergence is achieved by introducing control functions, by means of which the sequence of approximations is reorganized to become convergent for any given parameters.

The approach is based on the property of self-similarity between approximations. This property, for the family of endomorphisms representing the given approximation sequence, is a necessary condition for the motion in the vicinity of a fixed point. For the approximation sequence itself, this means a necessary condition for the fastest convergence $[42]$.

Let us stress the difference between the self-similarity of approximations and the functional self-similarity which is the basis of any renormalization-group approach $[46,60]$. In the latter case, one looks for a symmetry of the considered function $f(x)$ with respect to the scaling of the variable $x$, so that to find a relation $f(\lambda x)=B(\lambda, f(x))$. From such a relation, it is easy to get the renormalization group equation $x \partial f(x) / \partial x=\beta(f(x))$, where $\beta(f) \equiv \lim _{\lambda \rightarrow 1} \partial B(\lambda, f) / \partial \lambda$ is called the renormalization group function. When the above relation is exact, one speaks of an exact renormalization group. However, in many cases such relations can be derived only approximately. As is clear from comparing this standard renormalization group technique with our approach, we do not consider the scaling of variables, but instead we are analysing a kind of scaling of approximation numbers. This what makes our approach principally different from any variant of the renormalization group techniques.

The property of the self-similarity of approximations permits us to reformulate perturbation theory to the language of dynamical theory and optimal control theory. Such 
a reformulation allows us to resort to powerful methods of these theories in order to give a logical foundation for the whole approach. Thus, it immediately becomes clear that control functions are to be defined by the fixed-point equations. Then, considering the motion near a fixed point, we find nontrivial corrections essentially improving the accuracy of approximations. And, what is probably the most important, we can use the stability analysis in order to check the stability of the procedure and, respectively, the convergence of the approximation sequence. This is based on the analysis of the local multipliers that are directly related to the local Lyapunov exponents.

What is especially valuable is that the stability analysis allows us to decide when we can trust to the results of calculations, even if we do not know exact answers. This also permits us do choose between several ways of calculations, differring by initial approximations. The general guide is always the same: We must select the most stable procedure.

In the present paper, we have considered a variety of examples for which accurate numerical data are available. Then, directly comparing the latter with our results, we could explicitly demonstrate that the method does work. We think that such an explicit demonstration is a necessary step before applying the method to more complicated problems for which no numerical data are known.

\section{Appendix A}

The integral (77) has the following properties that we have used in the process of calculations:

$$
\begin{gathered}
I_{n s}^{l}(\nu)=I_{s n}^{l}(\nu), \quad I_{n s}^{l}(0)=\frac{\Gamma(n+l+3 / 2)}{n !} \delta_{n s}, \\
I_{00}^{l}(\nu)=\Gamma\left(l+\frac{3}{2}+\nu\right),
\end{gathered}
$$

where

$$
\begin{gathered}
\varphi_{n}(x) \equiv \frac{\Gamma(n+x)}{\Gamma(x)}=x(1+x)(2+x) \ldots(n-1+x), \\
\varphi_{0}(x)=1, \quad \varphi_{1}(x)=x, \quad x \in(-\infty,+\infty), \\
\Gamma(-x) \Gamma(x)=-\frac{\pi}{x \sin (\pi x)} .
\end{gathered}
$$


Also,

$I_{n s}^{l}(1)=\left(2 n+l+\frac{3}{2}\right) \frac{\Gamma(n+l+3 / 2)}{n !} \delta_{s n}-\frac{\Gamma(n+l+3 / 2)}{(n-1) !} \delta_{s, n-1}-\frac{\Gamma(n+l+5 / 2)}{n !} \delta_{s, n+1}$.

In general, integral (77) can be presented as the sum

$$
I_{n s}^{l}(\nu)=\frac{\Gamma(n+l+3 / 2)}{s !} \sum_{p=0}^{n} \frac{(-1)^{p} \Gamma(p+l+3 / 2+\nu)}{p !(n-p) ! \Gamma(p+l+3 / 2)} \varphi_{s}(-p-\nu) .
$$

Employing these properties, the coefficients in the sequence (76), for the case $n=0$, can be simplified to

$$
\begin{gathered}
A_{0 l}(\nu)=\frac{\Gamma(l+3 / 2+\nu)}{(l+3 / 2) \Gamma(l+3 / 2)}, \quad A_{0 l}(1)=1, \\
B_{0 l}(\nu)=\frac{\nu \Gamma(l+3 / 2+\nu)}{(l+3 / 2) \Gamma(l+3 / 2)}, \quad B_{0 l}(1)=1, \\
C_{0 l}(\nu)=\frac{\Gamma^{2}(l+3 / 2+\nu)}{(l+3 / 2) \Gamma(l+3 / 2)} \sum_{p=1}^{\infty} \frac{\varphi_{p}^{2}(-\nu)}{p p ! \Gamma(p+l+3 / 2)} .
\end{gathered}
$$

The value $\Delta(n, l)$ entering Eq. (78), for $n=0$, writes as

$$
\Delta(0, l)=\frac{1}{8}-\frac{\Gamma(l+5 / 2)}{2 \nu^{2}} \sum_{p=1}^{\infty} \frac{\varphi_{p}^{2}(-\nu)}{p p ! \Gamma(p+l+3 / 2)} .
$$

\section{Appendix B}

The integral (86), by employing the replica trick

$$
\ln t=\lim _{\nu \rightarrow 0} \frac{t^{\nu}-1}{\nu}
$$

can be expressed through the limit

$$
I_{n s}^{l}=\lim _{\nu \rightarrow 0} \frac{I_{n s}^{l}(\nu)-I_{n s}^{l}(0)}{\nu}
$$

involving the integral (77). Taking this limit, we meet the expansion

$$
\frac{\Gamma(x+\nu)}{\Gamma(x)} \simeq 1+\psi(x) \nu \quad(\nu \rightarrow 0)
$$


in which

$$
\begin{aligned}
\psi(x) \equiv \frac{d}{d x} \ln \Gamma(x) & =\sum_{p=0}^{\infty}\left(\frac{1}{p+1}-\frac{1}{p+x}\right)-C=\sum_{p=1}^{\infty} \frac{x}{p(p+x)}-\frac{1}{x}-C, \\
C & \equiv \lim _{n \rightarrow \infty}\left(\sum_{p=1}^{n} \frac{1}{p}-\ln n\right)=0.577216 .
\end{aligned}
$$

For $n$ integer, one has

$$
\begin{gathered}
\psi(n+1)=\sum_{p=1}^{n} \frac{1}{p}+\psi(1), \quad \psi(1)=-C, \\
\psi\left(n+\frac{1}{2}\right)=\sum_{p=1}^{n} \frac{2}{2 p-1}-2 \ln 2-C, \quad \psi\left(\frac{3}{2}\right)=2-\ln 4-C=0.036490 .
\end{gathered}
$$

In the course of taking the limit $\nu \rightarrow 0$, we also need to consider the corresponding asymptotic behaviour of the function $\varphi_{n}(-p-\nu)$ defined in Appendix A. This asymptotic behaviour is

$$
\varphi_{n}(-p-\nu) \simeq\left\{\begin{array}{cc}
(-1)^{p+1}(n-p-1) ! p ! \nu & (0 \leq p \leq n-1) \\
(-1)^{n} n !\{1+[\psi(n+1)-\psi(1)] \nu\} & (p=n),
\end{array}\right.
$$

as $\nu \rightarrow 0$. Using these equalities, for the integral (86), in the case of $n=s$, we find

$$
I_{n n}^{l}=\frac{\Gamma(n+l+3 / 2)}{n !} \psi\left(n+l+\frac{3}{2}\right) .
$$

When $n<s$, then

$$
I_{n s}^{l}=-\frac{\Gamma(n+l+3 / 2)}{s !} \sum_{p=0}^{n} \frac{(s-p-1) !}{(n-p) !} \quad(n<s)
$$

while in the opposite case,

$$
I_{n s}^{l}=-\frac{\Gamma(n+l+3 / 2)}{s !}\left[\sum_{p=0}^{s-1} \frac{(s-p-1) !}{(n-p) !}+\sum_{p=0}^{n-s} \frac{(-1)^{p}}{p !(n-p-s) !} \psi_{p s}^{l}\right] \quad(n>s),
$$

where

$$
\psi_{p s}^{l} \equiv \psi(p+1)-\psi(p+s+1)-\psi\left(p+s+l+\frac{3}{2}\right)
$$

We also have

$$
I_{n p}^{l}=\lim _{\nu \rightarrow 0} \frac{1}{\nu} I_{n p}^{l}(\nu) \quad(n \neq p)
$$




$$
I_{n, n-1}^{l}=-\frac{\Gamma(n+l+1 / 2)}{(n-1) !}, \quad I_{n, n+1}^{l}=-\frac{\Gamma(n+l+3 / 2)}{n !}, \quad I_{0 s}^{l}=-\frac{\Gamma(l+3 / 2)}{s !},
$$

In this way, we can calculate any coefficient

$$
C_{n l}=\lim _{\nu \rightarrow 0} \frac{1}{\nu^{2}} C_{n l}(\nu),
$$

in the sequence (85). In particular, for $n=0$, we get

$$
C_{0 l}=\frac{\Gamma(l+3 / 2)}{l+3 / 2} \sum_{p=1}^{\infty} \frac{(p-1) !}{p^{2} \Gamma(p+l+3 / 2)} .
$$

\section{Appendix $\mathrm{C}$}

The integral (102) can be presented as the limit

$$
J_{n s}^{l}=\lim _{\nu \rightarrow 0} \frac{J_{n s}^{l}(\nu)-J_{n s}^{l}(0)}{\nu}
$$

involving the integral

$$
J_{n s}^{l}(\nu)=\int_{0}^{\infty} t^{2 l+2+\nu} e^{-t} L_{n}^{2 l+1}(t) L_{s}^{2 l+1}(t) d t .
$$

Substituting in the latter the Laguerre polynomials, we come to the expressions similar to those in Appendix A. In this way,

$$
J_{n n}^{l}(\nu)=\frac{\Gamma(n+2 l+2)}{n !} \sum_{p=0}^{n} \frac{(-1)^{p} \Gamma(p+2 l+3+\nu)}{p !(n-p) ! \Gamma(p+2 l+2)} \varphi_{n}(-p-1-\nu),
$$

where $\varphi_{n}(x)$ is the same function as in Appendix A. Also we find

$$
J_{n n}^{l}(0)=2(n+l+1) \frac{(n+2 l+1) !}{n !}, \quad J_{n n}^{l}(-1)=\frac{(n+2 l+1) !}{n !} .
$$

Taking the limit $\nu \rightarrow 0$, we use the asymptotic equalities

$$
\varphi_{n}(-p-1-\nu) \simeq(-1)^{p}(n-p-2) !(p+1) ! \nu
$$

for $0 \leq p \leq n-2$, and

$$
\varphi_{n}(-p-1-\nu) \simeq(-1)^{n}(p+1) !\{1+[\psi(p+2)-\psi(p-n+2)] \nu\}
$$


for the case of $p=n-1, n$. Here the function $\psi(x)$ is defined in Appendix B. With these properties we obtain the expression

$$
J_{n n}^{l}=\frac{(n+2 l+1) !}{n !}[2 n+1+2(n+l+1) \psi(n+2 l+2)]
$$

defining the coefficients $D_{n l}$ in Eq. (103),

$$
D_{n l}=\frac{n ! J_{n n}^{l}}{2(n+l+1)(n+2 l+1) !} .
$$




\section{References}

[1] P.M. Stevenson, Phys. Rev. D 30 (1984), 1712.

[2] P.M. Stevenson, Phys. Rev. D 32 (1985), 1389.

[3] P.M. Stevenson and I. Roditi, Phys. Rev. D 33 (1986), 2305.

[4] P.M. Stevenson, Z. Phys. C 35 (1987), 467.

[5] P.M. Stevenson, B. Alles, and R. Tarrach, Phys. Rev. D 35 (1987), 2407.

[6] S.K. Gandhi and M.B. Pinto, Phys. Rev. D 49, (1994) 4258.

[7] G.A. Hajj and P.M. Stevenson, Phys. Rev. D 37 (1988), 413.

[8] H. Haugerud and F. Ravndal, Phys. Rev. D 43 (1991), 2736.

[9] R.L. Hall, J. Phys. A 25 (1992), 4459.

[10] R.L. Hall, J. Phys. A 28 (1995), 1771.

[11] R.L. Hall and N. Saad, J. Phys. A 29 (1996), 2127.

[12] R.L. Hall and N. Saad, J. Phys. A 29 (1996), 5657.

[13] R.L. Hall and N. Saad, J. Math. Phys. 38 (1997), 4909.

[14] I. Stancu and P.M. Stevenson, Phys. Rev. D 42 (1990), 2710.

[15] I. Stancu, Phys. Rev. D 43 (1991), 1283.

[16] A. Okopińska, Phys. Rev. D 35 (1987), 1835.

[17] A. Okopińska, Ann. Phys. (N.Y.) 228 (1993), 19.

[18] T. Hatsuda, T. Kunihiro, and T. Tanaka, Phys. Rev. Lett. 78 (1997), 3229.

[19] T. Kunihiro, Phys. Rev. D 57 (1998), 2035. 
[20] V.I. Yukalov, Mosc. Univ. Phys. Bull. 31 (1976), 10.

[21] V.I. Yukalov, Theor. Math. Phys. 28 (1976), 652.

[22] V.I. Yukalov, Physica A 89 (1977), 363.

[23] W.E. Caswell, Ann. Phys. (N.Y.) 123 (1979), 153.

[24] I. Halliday and P. Suranyi, Phys. Rev. D 21 (1980), 1529.

[25] G. Grunberg, Phys. Lett. B 95 (1980), 70.

[26] I.K. Dmitrieva and G.I. Plindov, Phys. Lett. A 79 (1980), 47.

[27] P.M. Stevenson, Phys. Rev. D 23 (1981), 2916.

[28] P.M. Stevenson, Phys. Rev. D 24 (1981), 1622.

[29] J. Killingbeck, J. Phys. A 14 (1981), 1005.

[30] I.D. Feranchuk, L.I. Komarov, I.V. Nichipor, and A.P. Ulyanenkov, Ann. Phys. (N.Y.) 238 (1995), 370.

[31] M. Dineykhan, G.V. Efimov, G. Gandbold, and S.N. Nedelko, "Oscillator Representation in Quantum Physics", Springer, Berlin, 1995.

[32] S.A. Pernice and G. Oleaga, Phys. Rev. D 57 (1998), 1144.

[33] J.H. Field, Ann. Phys. (N.Y.) 226 (1993), 209.

[34] S.J. Brodsky, G.P. Lepage, and P.B. Mackenzie, Phys. Rev. D 28 (1983), 228.

[35] A. Duncan and H.F. Jones, Phys. Rev. D 47 (1993), 2560.

[36] C.M. Bender, A . Duncan, and H.F. Jones, Phys. Rev. D 49 (1994), 4219.

[37] R. Guida, K. Konishi and H. Suzuki, Ann. Phys. (N.Y.) 241 (1995), 152.

[38] V.I. Yukalov, Int. J. Mod. Phys. B 3 (1989), 1691. 
[39] V.I. Yukalov, Physica A 167 (1990), 833.

[40] V.I. Yukalov, Phys. Rev. A 42 (1990), 3324.

[41] V.I. Yukalov, J. Math. Phys. 32 (1991), 1235.

[42] V.I. Yukalov, J. Math. Phys. 33 (1992), 3994.

[43] V.I. Yukalov and E.P. Yukalova, Physica A 198 (1993), 573.

[44] V.I. Yukalov and E.P. Yukalova, Physica A 206 (1994), 553.

[45] V.I. Yukalov and E.P. Yukalova, Physica A 225 (1996), 336.

[46] J. Zinn-Justin, "Quantum Field Theory and Critical Phenomena", Clarendon, Oxford, 1996.

[47] M.C. Irwin, "Smooth Dynamical Systems", Academic, London, 1980.

[48] J.A. Walker, "Dynamical Systems and Evolution Equations", Plenum, New York, 1980.

[49] D.J. Rudolf, "Fundamentals of Measurable Dynamics", Clarendon, Oxford, 1990.

[50] C. Robinson, "Dynamical Systems", CRC, Boca Raton, 1995.

[51] H.J. Stetter, "Analysis of Discretization Methods for Ordinary Differential Equations", Springer, Berlin, 1973.

[52] E.C. Titchmarh, "Eigenfunction Expansions Associated with Second-Order Differential Equations", Vol. 2, Clarendon, Oxford, 1958.

[53] S. Bell, R. Davidson, and P. Warsop, J. Phys. B 3 (1970), 123.

[54] D. Secrest, K. Cashion, and J. Hirschfelder, J. Chem. Phys. 37 (1962), 830.

[55] T. Imbo, A. Pagnamenta, and U. Sukhatme, Phys. Rev. D 29 (1984), 1669. 
[56] R.L. Hall, Phys. Rev. D 37 (1988), 540.

[57] R.L. Hall, Phys. Rev. A 39 (1989), 5500.

[58] R.L. Hall, Phys. Rev. A 51 (1995), 3499.

[59] C. Quigg and J. Rosner, Phys. Rep. 56 (1979), 167.

[60] N.N. Bogolubov and D.V. Shirkov, "Quantum Fields", Benjamin, London, 1983. 


\section{Table captions}

Table I. The accuracy of different approximations for the ground-state energy $e(0,0)$, for various povers $\nu$ of the potential: $\varepsilon_{1}$ and $\varepsilon_{2}$ are the percentage errors of the renormalized expressions in Eq. (79); $\varepsilon_{2}^{*}$ is the error of the self-similar approximant (80); and $\varepsilon_{W K B}$ is the error of the quasiclassical approximation (73).

Table II. The accuracy of approximations for several energy levels $e(0, l)$ of the logarithmic potential: $\varepsilon_{1}$ and $\varepsilon_{2}$ are the percentage errors of the renormalized expressions (89); $\varepsilon_{2}^{*}$ is the error of the self-similar approximant (90); and $\varepsilon_{W K B}$ is the error of the quasiclassical approximation (83). 
Table I

\begin{tabular}{|c|c|c|c|c|c|}
\hline$\nu$ & $e(0,0)$ & $\varepsilon_{1}(\%)$ & $\varepsilon_{2}(\%)$ & $\varepsilon_{2}^{*}(\%)$ & $\varepsilon_{W K B}(\%)$ \\
\hline 0.15 & 1.2653 & 0.26 & 0.05 & -0.49 & -0.73 \\
0.50 & 1.5961 & 0.44 & 0.07 & -0.02 & -1.2 \\
0.75 & 1.7450 & 0.39 & 0.06 & 0.08 & -1.0 \\
1.5 & 2.0121 & 0.08 & 0.01 & 0.04 & -0.26 \\
2 & 2.1213 & 0 & 0 & 0 & 0 \\
3 & 2.2765 & 0.33 & -0.05 & 0.17 & -0.27 \\
4 & 2.3936 & 1.3 & -0.43 & 0.63 & -1.3 \\
5 & 2.4924 & 2.6 & -1.6 & 1.2 & -2.6 \\
6 & 2.5797 & 4.3 & -4.0 & 1.6 & -4.1 \\
8 & 2.7315 & 8.5 & -16 & 1.0 & -7.1 \\
10 & 2.8616 & 13 & -50 & -4.3 & -9.9 \\
\hline
\end{tabular}

Table II

\begin{tabular}{|c|c|c|c|c|c|}
\hline$l$ & $e(0, l)$ & $\varepsilon_{1}(\%)$ & $\varepsilon_{2}(\%)$ & $\varepsilon_{2}^{*}(\%)$ & $\varepsilon_{W K B}(\%)$ \\
\hline 0 & 0.52215 & 2.2 & 0.48 & 0.37 & -6.4 \\
1 & 0.82150 & 0.81 & 0.10 & -0.36 & -9.4 \\
2 & 1.0075 & 0.47 & 0.05 & -0.38 & -9.4 \\
3 & 1.1430 & 0.31 & 0.01 & -0.36 & -9.2 \\
4 & 1.2495 & 0.22 & -0.002 & -0.33 & -8.9 \\
\hline
\end{tabular}

